\title{
PRODUÇÃO DE ADSORVENTE A PARTIR DE SABUGO DE MILHO PARA REMOÇÃO DE FENOL EM ESCALAS LABORATORIAL E INDUSTRIAL
}

\author{
P.D. ROCHA, A.S. FRANCA e L.S. OLIVEIRA \\ Universidade Federal de Minas Gerais, Departamento de Engenharia Mecânica \\ E-mail para contato: adriana@demec.ufmg.br
}

\begin{abstract}
RESUMO - O fenol aparece em diversos tipos de efluentes industriais, como refinarias de petróleo, tintas e papel. Tal composto é considerado um poluente prioritário por ser danoso aos organismos já em baixas concentrações e pode ser tóxico em níveis mais elevados. A adsorção por carvão ativado (CA) é o método de remoção mais utilizado. Devido à necessidade de um processo de regeneração do CA, existe um aumento dos custos. Portanto, busca-se adsorventes alternativos de baixo custo que apresentem capacidade de adsorção apropriada. Dessa forma, o presente trabalho objetivou desenvolver um adsorvente alternativo a base de sabugo de milho para remoção de fenol de solução aquosa. $\mathrm{O}$ sabugo foi submetido a seguinte tratamento: i) secagem a $105^{\circ} \mathrm{C}$ por $18 \mathrm{~h}$; ii) ativação com ácido fosfórico $(85 \%)$; iii) $2 \mathrm{~h}$ de carbonização em forno mufla a $500^{\circ} \mathrm{C}$; iv) lavagem com hidróxido de sódio até $\mathrm{pH}$ neutro e v) secagem a $105^{\circ} \mathrm{C}$ por $12 \mathrm{~h}$. O desempenho do CA produzido foi avaliado em ensaios de adsorção em batelada, para remoção do fenol de soluções aquosas, e ensaio de adsorção em coluna, visando posterior aplicação industrial. Foram realizados procedimentos de caracterização superficial, como análise em infravermelho (IV) e titulação de Boehm, objetivando a compreensão do mecanismo pelo qual o processo de adsorção ocorre. Os resultados obtidos demonstraram que o tratamento foi efetivo na produção de um CA seletivo para remoção de fenol.
\end{abstract}

\section{INTRODUÇÃO}

Fenóis aparecem em efluentes residuais de indústrias, como de conversão de carvão a altas temperaturas, refinarias de petróleo, resinas e outras, e são considerados poluentes prioritários, por serem tóxicos aos seres vivos. Dessa maneira, é necessário remover o fenol de efluentes industriais antes do lançamento destes em cursos d'água, e, em condições apropriadas, podem ser recuperados desses efluentes de maneira econômica. Entretanto, os melhores métodos para tratar esses efluentes são por oxidação química ou por adsorção (Bello et al., 2013). A adsorção por carvão ativado é o método mais frequentemente utilizado. Recentemente, houve um crescente aumento de pesquisas na literatura voltadas para o estudo de adsorção para remoção de fenol com CAs (Bello et al., 2013), em que o fenol permanece retido na superfície do carvão. A maior desvantagem desta técnica é a saturação do adsorvente, que torna necessária a regeneração do CA. O processo de regeneração promove perda de CA, eleva custos de operação do processo e diminui a capacidade de adsorção a cada ciclo de regeneração. Tais aspectos incentivaram a 


\section{9 a 22 de outubro de 2014 \\ Florianópolis/SC}

produção de adsorventes de baixo custo, que apresentam capacidade adequada de adsorção sem necessidade de regeneração (Oliveira e Franca, 2012). Estes são geralmente produzidos de resíduos agroindustriais, materiais disponíveis em grandes quantidades e renováveis, e.g., cascas de arroz, sabugo de milho e outros.

O objetivo deste trabalho foi produzir um CA usando sabugo de milho como precursor e usar o adsorvente produzido para remover fenol de soluções aquosas. Neste trabalho, avaliou-se o desempenho do adsorvente em processos de adsorção em batelada e contínuo (leito fixo).

\section{METODOLOGIA}

Para a produção do carvão, o sabugo de milho foi cominuído até 20 mesh. Foram adicionados $25 \mathrm{~mL}$ de $\mathrm{H}_{3} \mathrm{PO}_{4} 85 \%$ a $25 \mathrm{~g}$ de sabugo. Após homogeneização, a mistura foi mantida em forno mufla a $500^{\circ} \mathrm{C}$ por $2 \mathrm{~h}$, sob atmosfera de $\mathrm{N}_{2}$ à $500 \mathrm{~mL} / \mathrm{min}$. Após resfriamento, o CA foi lavado com $\mathrm{NaOH}$ a $60^{\circ} \mathrm{C}$ até a neutralização do $\mathrm{pH}$. Em seguida, foi mantido em estufa a $110^{\circ} \mathrm{C}$ por $12 \mathrm{~h}$. Para estudo da capacidade de adsorção do CA produzido, foram preparadas soluções de fenol, em concentrações variando de 100 a $500 \mathrm{mg} / \mathrm{L}$, e efetuados testes de adsorção em batelada. Em Erlenmeyer de $250 \mathrm{~mL}$, foram adicionados 1,5 g de CA e $150 \mathrm{~mL}$ de solução de fenol. Os Erlenmeyers foram agitados a $100 \mathrm{rpm}$ por $24 \mathrm{~h}$ em mesa agitadora. Foram coletadas alíquotas de $1 \mathrm{~mL}$, em tempos determinados durante a adsorção. As amostras foram analisadas por espectrofotometria UV-Vis em comprimento de onda $269 \mathrm{~nm}$ para determinar as concentrações de fenol em solução.

Os experimentos em leito fixo foram conduzidos em coluna cilíndrica de aço (d.i. $2,5 \mathrm{~cm}$ e h $10 \mathrm{~cm}$ ). $12 \mathrm{~g}$ de adsorvente foram colocados na coluna e soluções de fenol foram bombeadas pela coluna. Amostras foram coletadas na saída em diferentes intervalos de tempo e analisadas por espectrofotometria UV-Vis $(269 \mathrm{~nm})$. A operação foi cessada quando a concentração de fenol no efluente atingiu $99 \%$ da de entrada. A concentração de entrada e vazão foram variadas de 200 a $500 \mathrm{mg} / \mathrm{L}$ e de 18,3 a $33 \mathrm{~mL} / \mathrm{min}$, respectivamente. Para a caracterização do adsorvente, os grupos funcionais na superfície do carvão foram determinados pelo método de Boehm (Boehm, 1994), que se baseia na neutralização seletiva dos grupos funcionais da superfície do carvão ativado por titulação. A caracterização dos grupos funcionais também foi efetuada por espectroscopia no infravermelho médio (FTIR), 4000-600 $\mathrm{cm}^{-1}$, antes e após a adsorção. O equilíbrio de adsorção foi avaliado pelos modelos de equilíbrio de Langmuir e de Freundlich (Alves et al., 2013). O modelo de Langmuir pode ser descrito por:

$$
q_{e}=\frac{q_{o} K_{L} C_{e}}{1+K_{L} C_{e}}
$$

em que $q_{e}$ é a capacidade de adsorção no equíbrio; $q_{m}$ é a capacidade máxima de adsorção; e $K_{L}$ é uma constante. $\mathrm{O}$ modelo de Freundlich pode ser descrito por:

$$
\mathrm{q}_{\mathrm{e}}=\mathrm{K}_{\mathrm{F}} \mathrm{C}_{\mathrm{e}}^{1 / \mathrm{n}}
$$


em que $q_{e}$ é a capacidade de adsorção no equíbrio; e $K_{F}$ e $n$ são constantes empíricas. Os modelos utilizados para a descrição do processo de adsorção em coluna são apresentados na Tabela 1.

Tabela 1 - modelos utilizados para descrição do processo de adsorção em coluna

\begin{tabular}{|c|c|}
\hline Modelo & Equação \\
\hline Bohart-Adams & $\frac{C}{C_{i}}=\frac{\exp \left(K_{B A} C_{i} t\right)}{\left.\exp C_{i} t\right)+\exp \left(K_{B A} N_{o} z / U_{o}\right)-1}$ \\
\hline Yoon-Nelson & $\frac{q_{0}=N_{0} z S / m}{C_{i}}=\frac{1}{1+\exp \left[k_{Y N}\left(t_{50}-t\right)\right] \quad q_{0}=\frac{C_{i} Q t_{50}}{m}}$ \\
\hline Thomas & $\frac{C}{C_{i}}=\frac{1}{\left(1+\exp \left[\left(K_{T} / Q\right) \cdot\left(q_{o} \cdot m-C_{i} \cdot V_{e f}\right)\right]\right.}$ \\
\hline Dose-Response & $\frac{C}{C_{i}}=1-\frac{1}{1+(Q t / b)^{a}}$ \\
\hline$q_{0}=\frac{C_{i} b}{m}$
\end{tabular}

$\mathrm{C}\left(\mathrm{mg} \mathrm{L}^{-1}\right)$ : concentração de adsorvato no fluido na saída; $\mathrm{C}_{\mathrm{i}}\left(\mathrm{mg} \mathrm{L}^{-1}\right)$ : concentração de adsorvato no fluido na entrada da coluna; $\mathrm{N}_{\mathrm{o}}\left(\mathrm{mg} \mathrm{L}^{-1}\right)$ : capacidade de adsorção; $\mathrm{z}(\mathrm{cm})$ : profundidade do leito; $\mathrm{U}_{\mathrm{o}}\left(\mathrm{cm} \mathrm{min}^{-1}\right)$ : velocidade superficial; $\mathrm{S}\left(\mathrm{cm}^{2}\right)$ : seção transversal do leito; $\mathrm{t}_{50}$ : tempo para alcançar 50\% da ruptura; Q ( $\left.\mathrm{L} \min ^{-1}\right)$ : vazão de fluido através da coluna; $\mathrm{m}(\mathrm{g})$ : massa de adsorvente; e $\mathrm{V}_{\text {ef }}(\mathrm{L})$ : volume de efluente; as constantes remanescentes são parâmetros empíricos para cada modelo específico.

\section{RESULTADOS E DISCUSSÃO}

Os resultados obtidos para a titulação de Boehm são mostrados na Tabela 2.

Tabela 2 - quantificação aproximada de alguns grupos funcionais na superfície do CA produzido

\begin{tabular}{|c|l|r|r|}
\hline \multicolumn{2}{|c|}{ Titulação de Boehm } & mmol/g & \multicolumn{1}{c|}{$\%$} \\
\hline \multirow{3}{*}{ Grupos Ácidos } & Fenólicos & 2,9259 & 38,36 \\
\cline { 2 - 4 } & Carboxílicos & 2,1232 & 27,84 \\
\cline { 2 - 4 } & Lactônicos & 2,3045 & 30,22 \\
\hline \multicolumn{2}{|c|}{ Grupos Básicos } & 0,2733 & 3,58 \\
\hline
\end{tabular}


Verificou-se que os grupos funcionais presentes em maior quantidade na superfície do carvão foram os ácidos. A relação, em ordem decrescente, dos teores dos grupos ácidos foi de fenólicos > lactônicos > carboxílicos. A predominância de grupos fenólicos, carboxílicos e lactônicos foi relatada para outros adsorventes preparados com $\mathrm{H}_{3} \mathrm{PO}_{4}$ (Alves et al., 2013). Os espectros FTIR obtidos para o adsorvente podem ser observados na Figura 1.

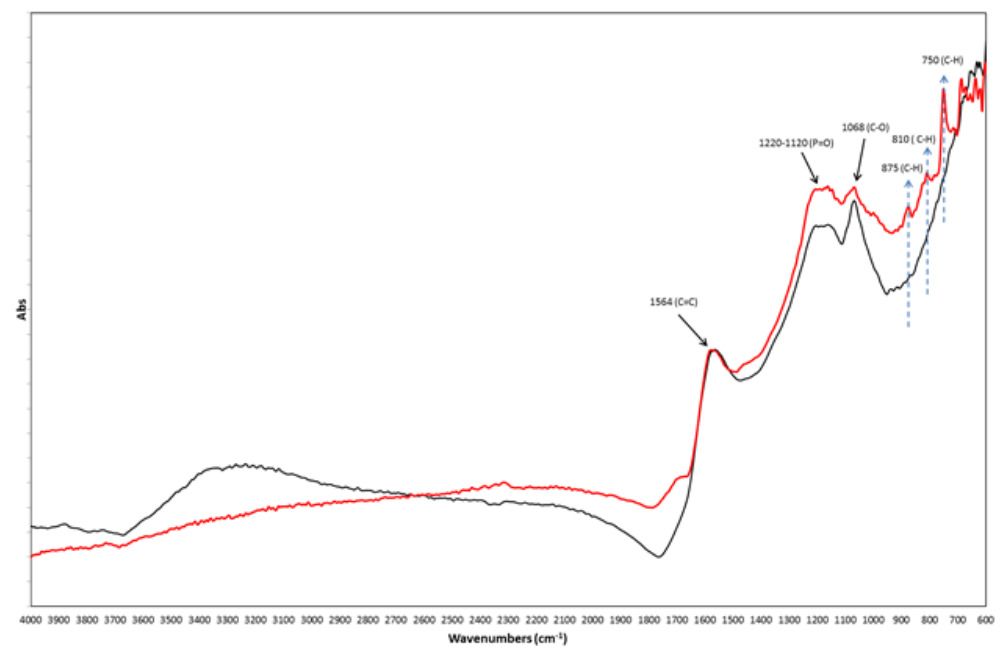

Figura 1 - Espectros FTIR para o CA antes (linha preta) e após adsorção do fenol (linha vermelha).

A banda observada na faixa de 3600 a $3000 \mathrm{~cm}^{-1}$ pode ser atribuída às vibrações de grupos hidroxílicos e/ou à água adsorvida (Kaouah et al., 2013). Quando os grupos hidroxílicos se referem à alcoóis e fenóis, também se verifica a presença de bandas de estiramento C-O na faixa de 1360 a $1000 \mathrm{~cm}^{-1}$ (Barbosa, 2008), como observado em $1068 \mathrm{~cm}^{-1}$, o que sugere a presença de grupos fenólicos. Por outro lado, após o processo de adsorção do fenol, a banda na faixa de 3600 a $3000 \mathrm{~cm}^{-1}$ desapareceu, de modo que a justificativa provável seja a interação do fenol com esse grupo funcional, uma vez que o pico característico em $1068 \mathrm{~cm}^{-1}$ diminuiu em intensidade, indicando associação com outro componente. A banda em $1691 \mathrm{~cm}^{-1}$ corresponde às vibrações de $\mathrm{C}=\mathrm{O}$, que podem aparecer ou não nos espectros em virtude da sobreposição de bandas relativas a $\mathrm{C}=\mathrm{C}$ de estruturas aromáticas localizadas em $1564 \mathrm{~cm}^{-1}$ (Kaouah et al., 2013). As bandas do estiramento $\mathrm{C}=\mathrm{C}$, indicando presença de estruturas aromáticas não sofreram grandes alterações após a adsorção de fenol. As bandas na faixa $1220-1120 \mathrm{~cm}^{-1}$ são características de carvões ativados com $\mathrm{H}_{3} \mathrm{PO}_{4}$ e estão associadas às vibrações de estiramento $\mathrm{P}=\mathrm{O}, \mathrm{P}-\mathrm{O}-\mathrm{C}$ e $\mathrm{P}=\mathrm{OOH}$ incorporadas por ligações de hidrogênio (Alves et al., 2013). Se o processo de adsorção for físico, a formação de multicamadas se torna mais evidenciada, sob aspecto da análise por FTIR, pelo surgimento e aumento da intensidade dos picos característicos de estruturas aromáticas. As bandas na região abaixo de $900 \mathrm{~cm}^{-1}$ são características de vibrações de dobramento entre os átomos de $\mathrm{C}-\mathrm{H}$ de compostos aromáticos (Barbosa, 2008). Quando a forma molecular do fenol é predominante, o mecanismo mais provável é baseado nas interações $\pi-\pi$ entre $o$ anel aromático do fenol e os elétrons $\pi$ deslocados na estrutura aromática do 
adsorvente. Contudo, ligações de hidrogênio com o grupamento fosfato e interações intermoleculares mais fracas constituem outros prováveis mecanismos de adsorção (Alves et al., 2013).

Na Tabela 3, mostram-se os dados para ajustes dos modelos de equilíbrio de adsorção de fenol para o carvão produzido. Percebe-se que o comportamento de adsorção de fenol do carvão produzido foi mais bem descrito pelo modelo de Freundlich, evidenciando processo de adsorção heterogêneo em multicamada.

Tabela 3 - parâmetros calculados para ajustes dos modelos de Freundlich e Langmuir

\begin{tabular}{|c|c|c|c|}
\hline Modelo & $\begin{array}{l}\text { Valores dos } \\
\text { parâmetros }\end{array}$ & $\mathrm{R}^{2}$ & RMS \\
\hline \multirow[t]{2}{*}{ Langmuir } & $\mathrm{K}_{\mathrm{L}}=0,0287$ & \multirow[t]{2}{*}{0,952} & \multirow[t]{2}{*}{6,387} \\
\hline & $Q_{\max }=52,254$ & & \\
\hline \multirow[t]{2}{*}{ Freundlich } & $n=1,937$ & \multirow[t]{2}{*}{0,988} & \multirow[t]{2}{*}{2,197} \\
\hline & $\mathrm{K}_{\mathrm{f}}=3,907$ & & \\
\hline
\end{tabular}

Os efeitos de concentração de entrada da solução de adsorbato e de vazão no processo de adsorção em leito fixo são representados nas curvas de ruptura nas Figuras 2 e 3, respectivamente. As curvas seguem o formato em "S", características de adsorvatos de tamanho molecular reduzido e de adsorventes com tamanho de partícula reduzido (Florido et al., 2010).

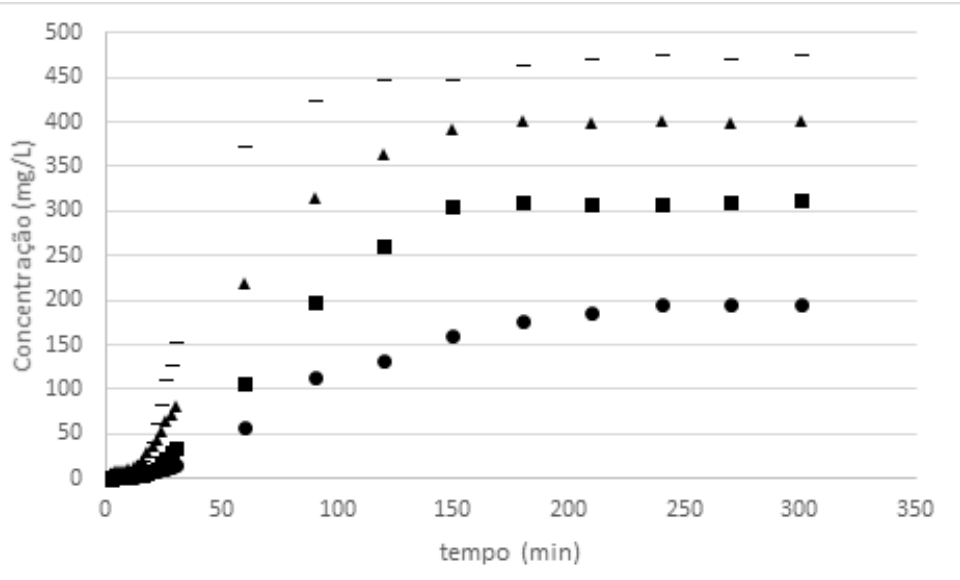

Figura 2 - Efeito de concentração de entrada na adsorção em leito fixo. • - $200 \mathrm{mg} / \mathrm{L}$, $\mathbf{-}$ $300 \mathrm{mg} / \mathrm{L}, \boldsymbol{\Delta}-400 \mathrm{mg} / \mathrm{L},-500 \mathrm{mg} / \mathrm{L}$. 


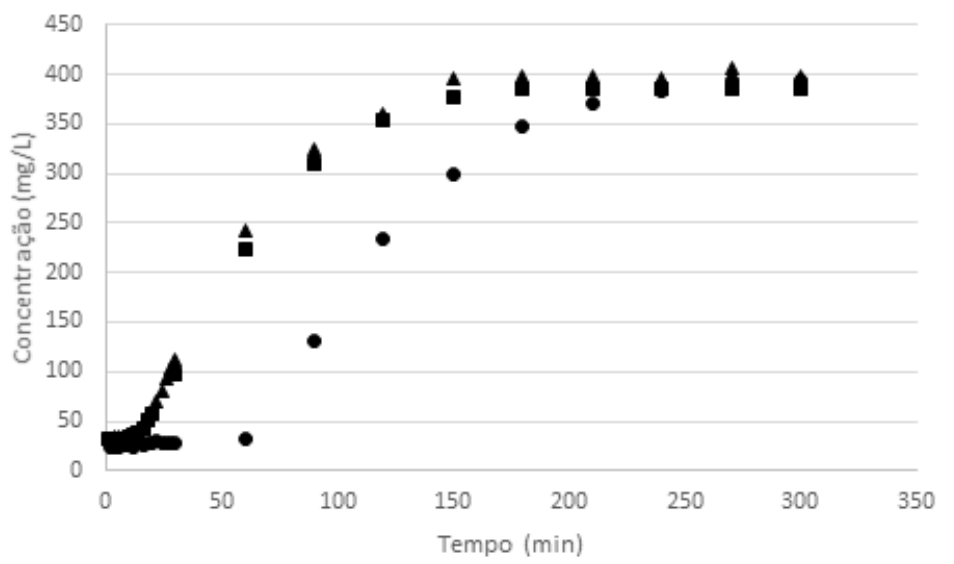

Figura 3 - Efeito da vazão na adsorção em leito fixo. $\bullet-18,315 \mathrm{~mL} / \mathrm{min}, \boldsymbol{\Delta}-25,641$ $\mathrm{mL} / \mathrm{min}, \mathbf{a}-32,967 \mathrm{~mL} / \mathrm{min}$.

Da Figura 2, percebe-se que o aumento na concentração de fenol na entrada da coluna causou uma redução dos tempos útil e de ruptura. Em outras palavras, a saturação do adsorvente foi alcançada mais rapidamente. $O$ aumento da concentração na entrada também elevou a inclinação da curva de ruptura (redução da zona de transferência de massa), reduzindo o volume de solução tratado antes da saturação. O decréscimo na concentração de entrada causou o aumento do tempo de ruptura. Os efeitos observados no comportamento da curva para aumento da vazão da solução de adsorvato (Figura 3) consistiram numa redução de volume tratado e no tempo de ruptura, com o tempo de vida útil do leito reduzido. Em concordância com este fator, para menores vazões, foram observados maiores tempos de vida útil e de ruptura. A inclinação da curva de ruptura aumentou com o aumento da vazão, indicando que a zona de transferência de massa foi reduzida em virtude dos efeitos de difusão intrapartícula mais acentuados. Por outro lado, Goyal et al. (2009) mostraram que sob um aumento da vazão acima de um dado valor, a taxa de adsorção decresce devido a uma redução significativa no tempo de residência do adsorbato na coluna. Observou-se aqui que um acréscimo da vazão acima de 32,97 $\mathrm{mL} / \mathrm{min}$ provavelmente não melhoraria o desempenho de adsorção, uma vez que não se percebe diferença significativa entre as curvas de ruptura para as vazões 25,64 e 32,97 $\mathrm{mL} / \mathrm{min}$. Os modelos de Bohart-Adams, Yoon-Nelson e Dose-Response foram ajustados aos dados experimentais e os respectivos parâmetros estimados por regressões linear e não linear. Esta última forneceu melhores resultados para todos os modelos e suas estimativas são as apresentadas e discutidas nesse trabalho.

Os resultados para ajustes dos modelos de curva de ruptura são apresentados na Tabela 4, juntamente com valores determinados experimentalmente. 
Tabela 4 - parâmetros calculados para os modelos de adsorção em coluna

\begin{tabular}{|c|c|c|c|c|c|c|c|c|}
\hline \multirow[t]{2}{*}{ Modelo } & \multirow[t]{2}{*}{ Parâmetros } & \multicolumn{4}{|c|}{$\mathrm{Ci}(\mathrm{mg} / \mathrm{L})[\mathrm{Q}=32,967 \mathrm{~mL} / \mathrm{min}]$} & \multicolumn{3}{|c|}{$\mathrm{Q}(\mathrm{L} / \mathrm{min})[\mathrm{Ci}=403 \mathrm{mg} / \mathrm{L}]$} \\
\hline & & 200 & 300 & 400 & 500 & 0,01832 & 0,02564 & 0,03297 \\
\hline \multirow{4}{*}{ Bohart-Adams } & $\mathrm{K} \times 10^{4}$ & 1,8 & 1,6 & 1,57 & 1,35 & 1,57 & 1,35 & 1,57 \\
\hline & $\mathrm{q}_{0}(\mathrm{mg} / \mathrm{g})$ & 52,79 & 63,31 & 67,48 & 68,00 & 37,49 & 52,48 & 67,47 \\
\hline & $\mathrm{R}^{2}$ & 0,988 & 0,997 & 0,995 & 0,978 & 0,9935 & 0,9941 & 0,9946 \\
\hline & RMS & 2,098 & 2,124 & 0,131 & 0,281 & 0,583 & 0,336 & 0,1305 \\
\hline \multirow{4}{*}{$\begin{array}{c}\text { Dose- } \\
\text { Response }\end{array}$} & $\mathrm{a}$ & 2,541 & 3,0442 & 2,498 & 2,54 & 3,549 & 2,49836 & 1,86 \\
\hline & $\mathrm{q}_{\mathrm{f}}(\mathrm{mg} / \mathrm{g})$ & 45,77 & 57,83 & 60,95 & 61,26 & 32,13 & 44,98 & 60,95 \\
\hline & $\mathrm{R}^{2}$ & 0,9979 & 0,9963 & 0,9982 & 0,993 & 0,9795 & 0,9896 & 0,9879 \\
\hline & RMS & 0,081 & 0,101 & 0,074 & 0,103 & 0,156 & 0,077 & 0,0741 \\
\hline \multirow{5}{*}{ Yoon-Nelson } & $\mathrm{k}$ & 0,036 & 0,0492 & 0,0544 & 0,08 & 0,028 & 0,046 & 0,0544 \\
\hline & $\mathrm{q}_{0}(\mathrm{mg} / \mathrm{g})$ & 52,30 & 62,87 & 66,73 & 67,64 & 37,07 & 51,90 & 66,73 \\
\hline & $\mathrm{t}_{50}(\min )$ & 95,188 & 78,663 & 60,267 & 45,772 & 114,458 & 65,887 & 60,267 \\
\hline & $\mathrm{R}^{2}$ & 0,9999 & 0,9998 & 0,9998 & 1 & 0,9935 & 0,9941 & 0,9946 \\
\hline & RMS & 2,098 & 2,124 & 0,131 & 0,281 & 0,583 & 0,336 & 0,131 \\
\hline \multirow{2}{*}{ Experimental } & $\mathrm{q}_{0}$ & 47,75 & 59,34 & 61,78 & 64,42 & 35,98 & 47,28 & 63,22 \\
\hline & $\mathrm{t}_{50}$ & 86,9 & 72 & 55,8 & 46,9 & 104,5 & 54,9 & 57,1 \\
\hline
\end{tabular}

O modelo de Bohart-Adams assume que a taxa de adsorção é proporcional à capacidade residual do sólido e à concentração do adsorbato. Na literatura, tal modelo foi proposto descrever somente a porção inicial da curva de ruptura (Han et al., 2009), entretanto, nesse trabalho, o modelo mostrou-se razoavelmente ajustável somente à porção final dos dados experimentais, comprovado pelos valores de RMS apresentados na Tabela 4. As discrepâncias podem ser atribuídas ao fenômeno da dispersão axial, que não é levado em conta no modelo, e que é mais pronunciado quando o gradiente de concentração de adsorbato entre a solução e o adsorvente diminui ao longo da coluna. A constante k estimada pela regressão não linear do modelo de Bohart-Adams apresenta uma pequena variação, mas não significativa com as variações da vazão e concentração da solução na entrada da coluna, indicativo de que o mecanismo controlador da adsorção esteja mais relacionado à cinética do que à transferência de massa ou difusão intrapartícula. O modelo de Yoon-Nelson apresentou capacidades de adsorção similares aos do modelo de Bohart-Adams, fato esperado uma vez que a forma matemática da equação é a mesma. O modelo que apresentou a melhor descrição dos dados experimentais foi o Dose-Response. Este modelo foi proposto para descrição de biossorção de metais pesados em colunas É comumente utilizado para descrever diferentes processos farmacológicos. Por outro lado, a natureza do modelo exclui qualquer outra análise de mecanismo envolvido na adsorção ao longo da coluna. 


\section{CONCLUSÃO}

O objetivo do trabalho foi avaliar o desempenho de adsorventes produzidos de sabugo de milho para a remoção de fenol de soluções aquosas. Dados de equilíbrio indicaram adsorção favorável e foram mais bem descritos pelo modelo de Freundlich, indicando adsorção heterogênea e em multicamadas. A adsorção em coluna foi mais bem descrita pelo modelo DoseResponse com relação aos modelos de Yoon-Nelson e de Bohart-Adams. O sabugo apresenta grande potencial como um adsorvente alternativo de baixo custo para remoção de compostos fenólicos para tratamento de efluentes.

\section{REFERÊNCIAS BIBLIOGRÁFICAS}

ALVES, C. C. O.; FRANCA, A. S.; OLIVEIRA, L. S. Removal of phenylalanine from aqueous solutions with thermo-chemically modified corn cobs as adsorbents. LWT - Food Sci. Technol., v. 51, p. 1-8, 2013.

BARBOSA, L. C. A. Espectroscopia no Infravermelho na caracterização de compostos orgânicos. Viçosa: Editora UFV, 189 p., 2008.

BELlo, I. A.; OLADIPO, M. A.; GIWA, A. A.; ADEOYE, D. O. Adsorptive Removal of Phenolics from Wastewater; A Review. Int. J. Basic Appl. Sci., v. 2, p. 79-90, 2013.

FLORIDO A.; VALDERRAMA C.; AREVALDO J. A.; CASAS I.; MARTINEZ M.; MIRALLES N. Application of two sites non-equilibrium sorption model for the removal of $\mathrm{Cu}$ (II) onto grape stalk wastes in a fixed-bed column. Chem. Eng. Sci., v. 156, p. 298-304, 2010.

GOYAL M.; BHAGAT M.; DHAWAN R. Removal of mercury from water by fixed bed activated carbon columns. J. Hazard. Mater., v. 171, p. 1009-1015, 2009.

HAN, R.; WANG, Y.; ZHAO, X.; WANG, Y.; XIE, F.; CHENG, J.; TANG, M. Adsorption of methylene blue by phoenix tree leaf powder in a fixed-bed column: experiments and prediction of breakthrough curves. Desalination, v. 245, p. 284-297, 2009.

KAOUAH, F.; BOUMAZA, S.; BERRAMA, T.; TRARI, M.; BENDJAM, Z. Preparation and characterization of activated carbon from wild olive cores (oleaster) by $\mathrm{H}_{3} \mathrm{PO}_{4}$ for the removal of Basic Red 46. J. Clean. Prod., v. 54, 296-306, 2013.

OLIVEIRA L. S.; FRANCA A. S. Low-Cost Adsorbents from Agri-Food Wastes. In: Encyclopedia of Food Science Research. New York: Nova Publishers, p.171-210, 2012. 